\title{
Faces of the scl norm ball
}

\author{
DANNY CALEGARI
}

Let $F=\pi_{1}(S)$ where $S$ is a compact, connected, oriented surface with $\chi(S)<0$ and nonempty boundary.

(1) The projective class of the chain $\partial S \in B_{1}^{H}(F)$ intersects the interior of a codimension one face $\pi_{S}$ of the unit ball in the stable commutator length norm on $B_{1}^{H}(F)$.

(2) The unique homogeneous quasimorphism on $F$ dual to $\pi_{S}$ (up to scale and elements of $\left.H^{1}(F)\right)$ is the rotation quasimorphism associated to the action of $\pi_{1}(S)$ on the ideal boundary of the hyperbolic plane, coming from a hyperbolic structure on $S$.

These facts follow from the fact that every homologically trivial 1-chain $C$ in $S$ rationally cobounds an immersed surface with a sufficiently large multiple of $\partial S$. This is true even if $S$ has no boundary.

20F65, 20J05; 20F67, 20F12, 55N35, 57M07

\section{Introduction}

An immersed loop in the plane might or might not bound an immersed disk, and if it does, the disk it bounds might not be unique. An immersed loop on a surface might not bound an immersed subsurface, but admit a finite cover which does bound an immersed subsurface - ie it might "virtually" bound an immersed surface. Most homologically trivial geodesics on hyperbolic surfaces with boundary do not even virtually bound an immersed surface. However, in this paper, we show that every homologically trivial geodesic in a closed hyperbolic surface $S$ virtually bounds an immersed surface, and every homologically trivial geodesic in a hyperbolic surface $S$ with boundary virtually cobounds an immersed surface together with a sufficiently large multiple of $\partial S$. This has implications for the structure of the (second) bounded cohomology of free and surface groups, as we explain in what follows.

Given a group $G$ and an element $g \in[G, G]$, the commutator length of $g$, denoted $\operatorname{cl}(g)$, is the smallest number of commutators in $G$ whose product is $g$, and the stable commutator length of $g$ is the $\operatorname{limit} \operatorname{scl}(g):=\lim _{n \rightarrow \infty} \operatorname{cl}\left(g^{n}\right) / n$. Geometrically, if $X$ 
is a space with $\pi_{1}(X)=G$ and $g$ is represented by a loop $\gamma$ in $X$, the commutator length of $g$ is the least genus of a surface mapping to $X$ whose boundary maps to $\gamma$. By minimizing number of triangles instead of genus, one can reinterpret $\mathrm{scl}$ as a kind of $L^{1}$ norm on relative (2-dimensional) homology. Technically, if $B_{1}(G ; \mathbb{R})$ denotes the vector space of real-valued (group) 1-boundaries (ie group 1-chains which are boundaries of group 2-chains; see Section 2.2), there is a well-defined scl pseudo-norm on $B_{1}(G ; \mathbb{R})$. The subspace on which scl vanishes always includes a subspace $H$ spanned by chains of the form $g^{n}-n g$ and $g-h g h^{-1}$, for $n \in \mathbb{Z}$ and $g, h \in G$, and therefore scl descends to a pseudo-norm on the quotient $B_{1}(G ; \mathbb{R}) / H$, which we abbreviate by $B_{1}^{H}(G ; \mathbb{R})$ or $B_{1}^{H}(G)$ or even $B_{1}^{H}$ in the sequel. In certain special cases (for example, when $G$ is a free group), scl defines an honest norm on $B_{1}^{H}(F)$, but we will not use this fact in the sequel. More precise definitions and background are given in Section 2.

Dual (in a certain sense) to the space $B_{1}^{H}(G ; \mathbb{R})$ with its scl pseudo-norm is the space $Q(G)$ of homogeneous quasimorphisms on $G$, ie functions $\phi: G \rightarrow \mathbb{R}$ for which there is a least real number $D(\phi)$ (called the defect) such that $\phi\left(g^{n}\right)=n \phi(g)$ for all $g \in G$ and $n \in \mathbb{Z}$, and $|\phi(g)+\phi(h)-\phi(g h)| \leq D(\phi)$ for all $g, h \in G$. The particular form of duality between scl and $Q(G)$ is called Bavard duality, which is the equality

$$
\operatorname{scl}\left(\sum t_{i} g_{i}\right)=\sup _{\phi \in Q(G)} \frac{\sum t_{i} \phi\left(g_{i}\right)}{2 D(\phi)}
$$

(see Section 2 for details).

The defining properties of a homogeneous quasimorphism can be thought of as an infinite family of linear equalities and inequalities depending on elements and pairs of elements in $G$. The $L^{1}-L^{\infty}$ duality between scl and $Q(G)$ means that computing scl is tantamount to solving an (infinite dimensional) linear programming problem in group homology (for an introduction to linear programming; see eg Dantzig [9]). In finite dimensions, $L^{1}$ and $L^{\infty}$ norms are piecewise linear functions, and their unit balls are rational convex polyhedra. Broadly speaking, the main discovery of Calegari [6] is that in free groups (and certain groups derived from free groups in simple ways), computing $\mathrm{scl}$ reduces to a finite dimensional linear programming problem, and therefore the unit ball of the scl pseudo-norm on $B_{1}^{H}(F ; \mathbb{R})$ is a rational convex polyhedron; ie for every finite dimensional rational vector subspace $V$ of $B_{1}(F ; \mathbb{R})$, the unit ball of the scl pseudo-norm restricted to $V$ is a finite-sided rational convex polyhedron (compare with the well-known example of the Gromov-Thurston norm on $\mathrm{H}_{2}$ of a 3-manifold; see Thurston [21]).

In a finite dimensional vector space, a rational convex polyhedron is characterized by its top dimensional faces - ie those which are codimension one in the ambient space. 
In an infinite dimensional vector space, a rational convex polyhedron need not have any faces of finite codimension at all. The codimension of a face of a convex polyhedron in an infinite dimensional vector space is the supremum of the codimensions of its intersections with finite dimensional subspaces. Top dimensional faces of the unit ball of the Gromov-Thurston norm on $\mathrm{H}_{2}$ of a 3-manifold have a great deal of topological significance (see eg Thurston [21], Kronheimer and Mrowka [14], Calegari [7] or Ozsváth and Szabó [18] for connections with the theories of taut foliations, SeibergWitten equations, quasigeodesic flows and Heegaard Floer homology respectively). It is therefore a natural question to ask whether the scl unit polyhedron in $B_{1}^{H}(F ; \mathbb{R})$ has any faces which are codimension one in $B_{1}^{H}(F ; \mathbb{R})$, and whether some of these faces have any geometric significance. Our first two main theorems answer these questions affirmatively.

Theorem A Let $F$ be a free group, and let $S$ be a compact, connected, orientable surface with $\chi(S)<0$ and $\pi_{1}(S)=F$. Let $\partial S \in B_{1}^{H}(F ; \mathbb{R})$ be the 1-chain represented by the boundary of $S$, thought of as a finite formal sum of conjugacy classes in $F$. Then the projective ray in $B_{1}^{H}(F ; \mathbb{R})$ spanned by $\partial S$ intersects the unit ball of the scl norm in the interior of a face of codimension one in $B_{1}^{H}(F ; \mathbb{R})$.

By Bavard duality, a face of the scl norm ball of codimension one is dual to a unique extremal homogeneous quasimorphism, up to elements of $H^{1}$ (which vanish identically on $B_{1}^{H}$ ). It turns out that we can give an explicit description of the extremal quasimorphisms dual to the "geometric" faces of the scl norm ball described in Theorem A.

If $S$ is a compact, connected, orientable surface with $\pi_{1}(S)=F$, then $S$ admits a hyperbolic structure with geodesic boundary. The hyperbolic structure and a choice of orientation determine a discrete, faithful representation $\rho: \pi_{1}(S) \rightarrow \operatorname{PSL}(2, \mathbb{R})$, unique up to conjugacy. Since $\pi_{1}(S)$ is free, this representation lifts to $\widetilde{\rho}: \pi_{1}(S) \rightarrow \widetilde{\operatorname{SL}}(2, \mathbb{R})$ where $\widetilde{\mathrm{SL}}(2, \mathbb{R})$ denotes the universal covering group of $\operatorname{PSL}(2, \mathbb{R})$. There is a unique continuous homogeneous quasimorphism on $\widehat{\mathrm{SL}}(2, \mathbb{R})$ (up to scale), called the rotation quasimorphism (discussed in detail in Section 3.3). This quasimorphism pulls back by $\tilde{\rho}$ to a homogeneous quasimorphism $\operatorname{rot}_{S}$ on $\pi_{1}(S)$, which is well-defined up to elements of $H^{1}(S ; \mathbb{Z})$. Up to scale, this turns out to be the homogeneous quasimorphism dual to the top dimensional face of the scl norm ball described above:

Theorem B Let $F$ be a free group, and let $S$ be a compact, connected, orientable surface with $\chi(S)<0$ and $\pi_{1}(S)=F$. Let $\pi_{S}$ be the face of the scl unit norm ball whose interior intersects the projective ray of the class $\partial S$. The face $\pi_{S}$ is dual to the extremal homogeneous quasimorphism $\operatorname{rot}_{S}$.

These theorems are both proved in Section 3. 
Theorem A shows how hyperbolic geometry and surface topology manifest in the abstract (bounded) cohomology of a free group. Theorem B is a kind of rigidity result, characterizing the rotation quasimorphism associated to a discrete, faithful representation of $\pi_{1}(S)$ into $\operatorname{PSL}(2, \mathbb{R})$ amongst all homogeneous quasimorphisms by the property that it is "extremal" for $\partial S$. In Section 3.5 we use these theorems to deduce a short proof of a relative version of rigidity theorems of Goldman [11] and Burger-Iozzi-Wienhard [3], that representations of surface groups into certain Lie groups of maximal Euler class are discrete (it should be stressed that $[11 ; 3]$ contain much more than the narrow result we reprove).

In light of Theorem A and Theorem B, it is natural to ask whether the projective class of every element $g \in[F, F]$ intersects the interior of a face of the scl norm ball of finite codimension. In fact, it turns out that this is not the case. We show by an explicit example (Example 3.23) that there are many elements $g \in[F, F]$ where $F$ has rank at least 4 , whose projective classes are contained in faces of the scl norm ball of infinite codimension.

The method of proof is of independent interest. We show that for any homologically trivial geodesic 1-manifold $\gamma$ in a hyperbolic surface $S$, there is a surface $T$ and an immersion $f: T, \partial T \rightarrow S, \gamma$ for which $f_{*}[\partial T]$ is taken to some multiple of $[\gamma]+n[\partial S]$ in $H_{1}$; ie the 1-cycle $\gamma+n \partial S$ "rationally bounds" an immersed surface. Note that this remains true even if $S$ is closed! Explicitly, the statement of the main technical theorem (proved in Section 3.4) is as follows:

Theorem $\mathbf{C}$ Let $S$ be a compact, connected orientable surface with $\chi(S)<0$ and $C=\sum r_{i} g_{i}$ a finite rational chain in $B_{1}^{H}\left(\pi_{1}(S)\right)$. Then for all sufficiently large rational numbers $R$ (depending on $C$ ), the geodesic 1-manifold in $S$ corresponding to the chain $R \partial S+\sum r_{i} g_{i}$ rationally bounds an immersed surface $f: T \rightarrow S$.

The connection with stable commutator length is as follows: from the main theorem of [6] it follows that in an oriented hyperbolic surface $S$ with boundary, a rational 1 -chain $C$ bounds an immersed surface if and only if $\operatorname{scl}(C)=\operatorname{rot}_{S}(C) / 2$, where $\operatorname{rot}_{S}$ is as above (this is Proposition 3.8 below). Hence Theorem $C$ implies that every chain $C$ in $B_{1}^{H}\left(\pi_{1}(S)\right)$ which is projectively close enough to $\partial S$ satisfies $\operatorname{scl}(C)=\operatorname{rot}_{S}(C) / 2$; Theorems A and B follow.

A number of additional corollaries are stated, including a generalization of the main theorem of [8]. Let $G$ be a graph of free or (closed, orientable) hyperbolic surface groups amalgamated over infinite cyclic subgroups, and let $A$ be a nonzero rational class in $H_{2}(G)$. Let $\left[S_{1}\right], \ldots,\left[S_{m}\right]$ be the fundamental classes in $H_{2}$ of the vertex subgroups which are closed surface groups. Then for all sufficiently big integers $n_{i}$, 
some multiple of the class $A+\sum_{i} n_{i} \cdot\left[S_{i}\right]$ in $H_{2}(G)$ is represented by an injective map from a closed hyperbolic surface group to $G$.

\section{Background}

\subsection{Definitions}

The following definition is standard; see Bavard [1] or Calegari [5, Section 2.1].

Definition 2.1 Let $G$ be a group, and $g \in[G, G]$. The commutator length of $g$, denoted $\operatorname{cl}(g)$, is the smallest number of commutators in $G$ whose product is $g$.

Topologically, if $X$ is a space with $\pi_{1}(X)=G$, and $\gamma: S^{1} \rightarrow X$ is a loop representing the conjugacy class of $g$, then $\operatorname{cl}(g)$ is the least genus of a compact oriented surface $S$ with one boundary component for which there is a map $f: S \rightarrow X$ with $\left.f\right|_{\partial S}$ in the free homotopy class of $\gamma$.

Definition 2.2 Let $G$ be a group, and $g \in[G, G]$. The stable commutator length of $g$, denoted $\operatorname{scl}(g)$, is the limit

$$
\operatorname{scl}(g)=\lim _{n \rightarrow \infty} \frac{\operatorname{cl}\left(g^{n}\right)}{n}
$$

Commutator length and stable commutator length can be extended to finite linear sums of groups elements as follows:

Definition 2.3 Let $G$ be a group and $g_{1}, g_{2}, \ldots, g_{m}$ elements of $G$ whose product is in $[G, G]$. Define

$$
\begin{aligned}
\operatorname{cl}\left(g_{1}+\cdots+g_{m}\right) & =\inf _{h_{i} \in G} \operatorname{cl}\left(g_{1} h_{1} g_{2} h_{1}^{-1} \cdots h_{m-1} g_{m} h_{m-1}^{-1}\right) \\
\operatorname{scl}\left(g_{1}+\cdots+g_{m}\right) & =\lim _{n \rightarrow \infty} \frac{\operatorname{cl}\left(g_{1}^{n}+\cdots+g_{m}^{n}\right)}{n} .
\end{aligned}
$$

A geometric interpretation of these numbers will be given in Section 2.3.

It is a fact that the limit in Definition 2.3 exists and satisfies $\operatorname{scl}\left(g^{n}+\sum g_{i}\right)=\operatorname{scl}(n g+$ $\left.\sum g_{i}\right)$ and $\operatorname{scl}\left(g+g^{-1}+\sum g_{i}\right)=\operatorname{scl}\left(\sum g_{i}\right)$ for all $g, g_{i} \in G$ and $n \in \mathbb{N}$. So it makes sense to define $\operatorname{scl}\left(\sum n_{i} g_{i}\right)=\operatorname{scl}\left(\sum g_{i}^{n_{i}}\right)$ for any $n_{i} \in \mathbb{Z}$ and $g_{i} \in G$. With this definition, it is immediate that $\mathrm{scl}$ is subadditive; ie

$$
\operatorname{scl}\left(\sum g_{i}+\sum h_{j}\right) \leq \operatorname{scl}\left(\sum g_{i}\right)+\operatorname{scl}\left(\sum h_{j}\right) .
$$


If we need to emphasize the group $G$ we denote $\mathrm{cl}$ and $\mathrm{scl}$ by $\mathrm{cl}_{G}$ and $\operatorname{scl}_{G}$ respectively. One may reduce the calculation of scl on finite sums to a calculation of $\mathrm{scl}$ on individual elements, by the following "addition lemma":

Lemma 2.4 (Addition Lemma) Let $g_{1}, \ldots, g_{m} \in G$ have infinite order. Let $H=$ $G * F_{m-1}$ where $F_{m-1}$ is freely generated by $x_{1}, x_{2}, \ldots, x_{m-1}$. Then

$$
\operatorname{scl}_{G}\left(g_{1}+\cdots+g_{m}\right)+\frac{m-1}{2}=\operatorname{scl}_{H}\left(g_{1} x_{1} g_{2} x_{1}^{-1} \cdots x_{m-1} g_{m} x_{m-1}^{-1}\right) .
$$

This follows from [5, Theorem 2.93] and induction. When the $g_{i}$ have finite order, the formula must be corrected (in a straightforward way).

\section{2 scl as a pseudo-norm}

It is convenient to use the language of homological algebra. Given a group $G$, one has the complex of real group chains $C_{*}(G ; \mathbb{R}), \partial$ whose homology is the real (group) homology of $G$; see Mac Lane [15, Chapter IV, Section 5]. A real (group) $n$-chain is a finite formal real linear combination of elements of $G^{n}$, so (for instance) a real (group) 1 -chain is just a finite formal real linear combination of elements of $G$. Denote the group of (real) 1 -boundaries by $B_{1}(G ; \mathbb{R})$, or $B_{1}(G)$ for short.

The properties of scl enumerated in Section 2.1 imply that the function scl is welldefined, linear and subadditive on finite integral group 1-boundaries and therefore admits a unique linear continuous extension to $B_{1}(G)$.

Moreover, scl vanishes on the subspace $H$ of $B_{1}$ spanned by chains of the form $g^{n}-n g$ and $g-h g h^{-1}$ for any $g, h \in G$ and $n \in \mathbb{Z}$. Thus scl defines a pseudo-norm on $B_{1}^{H}:=B_{1} / H$. See Calegari [5, Section 2.6] for proofs of these basic facts.

In [6] an algorithm is described to compute scl on elements of $B_{1}^{H}(F)$ where $F$ is a free group. The program scallop (source available at [4]) implements a polynomialtime version of this algorithm, described in [5, Sections 4.1.7-4.1.8]. At a number of points in this paper we make assertions about the value of scl on certain chains in $B_{1}^{H}(F)$; these assertions are justified using the program scallop.

Remark 2.5 In the final analysis, our main theorems do not depend logically on the computations carried out with the aid of scallop (also see Remark 3.12). Nevertheless, these computations were an essential part of the process by which these theorems were discovered. 


\subsection{Extremal surfaces}

The definition of stable commutator length can be reinterpreted in geometric terms. Let $X$ be a space and $\gamma_{1}, \ldots, \gamma_{m}: S^{1} \rightarrow X$ nontrivial free homotopy classes of loops in $X$. Let $f: S \rightarrow X$ be a surface for which there is a commutative diagram

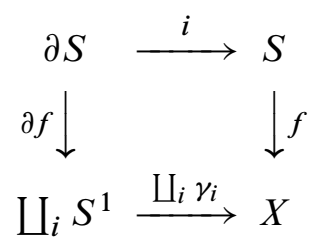

where $i: \partial S \rightarrow S$ is the inclusion map, and $\partial f_{*}[\partial S]=n\left[\coprod_{i} S^{1}\right]$ in $H_{1}$ for some $n$.

Define $\chi^{-}(S)$ to be the sum of Euler characteristic $\chi$ over all components of $S$ for which $\chi$ is nonpositive. Then there is an equality

$$
\operatorname{scl}_{G}\left(g_{1}+\cdots+g_{m}\right)=\inf _{S} \frac{-\chi^{-}(S)}{2 n}
$$

over all compact oriented surfaces $S$ as above, where $G=\pi_{1}(X)$, and $\gamma_{i}$ represents the conjugacy class of $g_{i}$. See Proposition 2.68 of [5] for a proof.

Definition 2.6 The chain $g_{1}+\cdots+g_{m}$ is said to rationally bound a surface $f: S \rightarrow X$ for which there is a commutative diagram as above. A surface with this property is extremal if every component of $S$ has negative Euler characteristic, and there is equality

$$
\operatorname{scl}\left(g_{1}+\cdots+g_{m}\right)=\frac{-\chi^{-}(S)}{2 n} .
$$

Extremal surfaces are $\pi_{1}$-injective [5, Proposition 2.96].

\subsection{Quasimorphisms}

Definition 2.7 Let $G$ be a group. A function $\phi: G \rightarrow \mathbb{R}$ is a homogeneous quasimorphism if it satisfies $\phi\left(g^{n}\right)=n \phi(g)$ for all $g \in G$ and $n \in \mathbb{Z}$, and if there is a least nonnegative real number $D(\phi)$ called the defect, such that for all $g, h \in G$ there is an inequality

$$
|\phi(g)+\phi(h)-\phi(g h)| \leq D(\phi) .
$$

The set of homogeneous quasimorphisms on a group $G$ is a real vector space and is denoted $Q(G)$. A homogeneous quasimorphism has defect 0 if and only if it is a homomorphism. Thus $H^{1}(G ; \mathbb{R})$ is a vector subspace of $Q(G)$. The defect $D$ defines 
a natural norm on $Q(G) / H^{1}(G ; \mathbb{R})$, making it into a Banach space. Any real-valued function on $G$ extends by linearity to define a 1 -cochain. There is an exact sequence

$$
0 \rightarrow H^{1}(G ; \mathbb{R}) \rightarrow Q(G) \stackrel{\delta}{\rightarrow} H_{b}^{2}(G ; \mathbb{R}) \rightarrow H^{2}(G ; \mathbb{R})
$$

where $\delta$ is the coboundary map on 1 -cochains, and $H_{b}^{*}$ denotes bounded (group) cohomology. See Bavard [1] or Calegari [5, Section 2.4] for an explanation of these facts and Gromov [12] for an introduction to bounded cohomology.

There is a duality between stable commutator length and quasimorphisms, called Bavard duality. For chains in $B_{1}^{H}(G)$, this takes the following form:

Theorem 2.8 (Bavard duality) Let $C=\sum t_{i} g_{i}$ be an element of $B_{1}^{H}(G ; \mathbb{R})$. Then there is an equality

$$
\operatorname{scl}(C)=\sup _{\phi \in Q(G) / H^{1}(G)} \frac{\sum t_{i} \phi\left(g_{i}\right)}{2 D(\phi)} .
$$

See Bavard [1] for a proof when $C$ is an element of $[G, G]$, or Calegari [5, Theorem 2.73] for the general case.

Definition 2.9 A quasimorphism $\phi \in Q(G)$ is extremal for a chain $C=\sum t_{i} g_{i} \in$ $B_{1}^{H}(G ; \mathbb{R})$ if there is equality

$$
\operatorname{scl}(C)=\frac{\sum t_{i} \phi\left(g_{i}\right)}{2 D(\phi)}
$$

Given a chain $C$, the set of extremal quasimorphisms for $C$ is a nonempty convex cone and is closed (away from 0 ) in the natural Banach space topology on $Q(G)$, as well as in the topology of termwise convergence in $\mathbb{R}^{G}$. See [5, Proposition 2.81] for a proof.

\section{Immersed surfaces}

\subsection{Doodles}

The question of which immersed loops in surfaces bound immersed subsurfaces is subtle and interesting and has fascinated many mathematicians (see eg Grothendieck [13, page 47]). An immersed loop in the plane which bounds an immersed disk necessarily has winding number \pm 1 , but not every loop with winding number \pm 1 bounds an immersed disk. See Figure 1. 

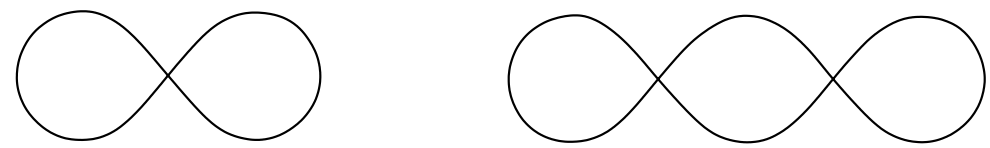

Figure 1: A figure 8 has winding number 0 , and therefore cannot bound an immersed disk. But a "double 8 " has winding number 1 , and does not bound an immersed disk either.

Blank [2] gave an algorithm to determine which immersed loops in the plane bound immersed disks; his algorithm was extended to other surfaces by Francis [10] and others, but the answer is not very illuminating: some curves bound immersed disks, some don't, and the reason is complicated. Other authors have studied the existence of branched immersions with prescribed boundary, which are much easier to construct.

Milnor [17] gave a well-known example of an immersed loop in the plane which bounds two different immersed disks. See Figure 2.

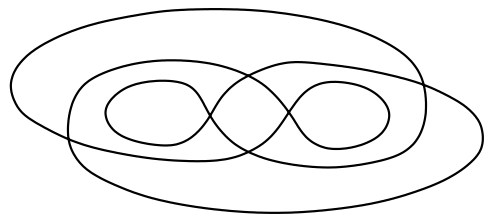

Figure 2: Milnor's "doodle" bounds an immersed disk in two inequivalent ways.

On a hyperbolic surface $S$, every homotopy class of essential loop contains a canonical geodesic representative. One can therefore ask which conjugacy classes in $\pi_{1}(S)$ are represented by geodesics which bound immersed surfaces. The answer turns out to be independent of the choice of hyperbolic structure on $S$, and therefore in principle is a purely "algebraic" problem.

One subtle aspect of the problem is illustrated by the example in Figure 3. This shows an immersed loop $\gamma$ (in the isotopy class of a geodesic) in a genus 2 punctured surface which does not bound an immersed surface, but which "virtually" bounds an immersed surface: there is an immersed surface with two boundary components, each of which wraps positively once around $\gamma$.

One is therefore led to study the following question. If $S$ is an orientable hyperbolic surface, which conjugacy classes $g$ in $\pi_{1}(S)$ are represented by geodesics which virtually bound immersed surfaces? That is, when is there an immersed surface in $S$, all of whose boundary components wrap positively around the geodesic representative of $g$ ? Only a homologically trivial loop virtually bounds a surface at all, so we restrict 


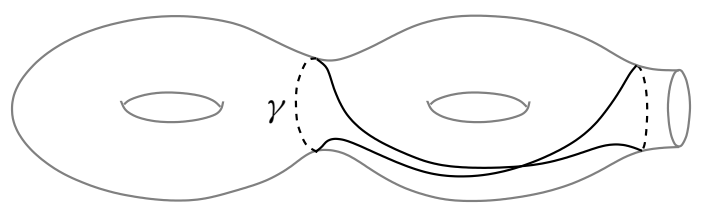

Figure 3: The loop $\gamma$ does not bound an immersed surface, but two copies of $\gamma$ do.

attention to $g$ in the commutator subgroup. It turns out that we can give a complete and somewhat surprising answer to this question. If $S$ is closed, then every homologically trivial geodesic virtually bounds an immersed surface. If $S$ has (geodesic) boundary, then a homologically trivial geodesic corresponding to a conjugacy class $g$ virtually bounds an immersed surface if and only if the projective class of $g$, thought of as a 1-boundary, intersects a certain top dimensional face of the unit ball in the scl pseudo-norm on $B_{1}^{H}\left(\pi_{1}(S)\right)$. This is explained in the remainder of this section.

\subsection{Positive immersed surfaces}

Definition 3.1 An immersion $f: T \rightarrow S$ between oriented surfaces is positive if it is orientation-preserving.

Definition 3.2 Let $\gamma: \bigsqcup_{i} S^{1} \rightarrow S$ be an immersed 1-manifold in $S$. The 1-manifold $\gamma$ bounds a (positive) immersion $f: T \rightarrow S$ if there is a commutative diagram

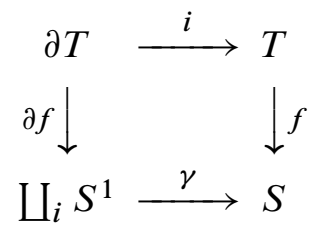

for which $\partial f: \partial T \rightarrow \coprod_{i} S^{1}$ is an orientation-preserving homeomorphism. The 1manifold $\gamma$ rationally bounds a positive immersion $f: T \rightarrow S$ if there is some integer $n$ and a commutative diagram as above, for which $\partial f: \partial T \rightarrow \coprod_{i} S^{1}$ is a positive immersion (ie an orientation-preserving covering map) such that $\partial f_{*}[\partial T]=n\left[\bigsqcup_{i} S^{1}\right]$ in $H_{1}$.

Compare with Definition 2.6.

We are concerned in the sequel with the case that $S$ is compact, connected and oriented, possibly with boundary and satisfying $\chi(S)<0$. The surface $S$ admits a (nonunique) hyperbolic structure with totally geodesic boundary; we fix such a structure. Let 
$C=\sum n_{i} g_{i}$ be a chain in $B_{1}^{H}\left(\pi_{1}(S) ; \mathbb{R}\right)$ where the $n_{i}$ are integers, and the $g_{i}$ are primitive.

For each $i$, let $\gamma_{i}: S^{1} \rightarrow S$ be a geodesic loop corresponding to the conjugacy class of $g_{i}$, and let $\gamma: \bigsqcup_{i} S^{1} \rightarrow S$ be the union of the $\gamma_{i}$. We say that $C$ rationally bounds a positive immersed surface if there is an integer $n$ and a positive immersion $f: T \rightarrow S$ as in Definition 3.2 for which $\partial f_{*}[\partial T]=n \cdot\left(\sum_{i} n_{i}\left[S_{i}^{1}\right]\right)$ in $H_{1}$, where $\gamma_{i}: S_{i}^{1} \rightarrow S$.

Lemma 3.3 Suppose chains $C_{1}, C_{2}$ rationally bound positive immersed surfaces. Then $C_{1}+C_{2}$ rationally bounds a positive immersed surface.

Proof For "generic" chains $C_{1}$ and $C_{2}$ there is nothing to prove: the disjoint union of two immersed surfaces is an immersed surface. The issue is that there might be a conjugacy class $g$ in the support of both $C_{1}$ and $C_{2}$ whose coefficients have different signs. Let $\gamma$ be the geodesic in the free homotopy class corresponding to $g$. Positive immersed surfaces with rational boundary $C_{1}$ and $C_{2}$ might have boundary components mapping to $\gamma$ with different degrees. The following claim shows that we can construct suitable covers of these immersed surfaces such that the boundary components mapping to $\gamma$ can be glued up.

Claim Let $S$ be a connected, oriented surface with $\chi(S)<0$ and genus at least 1. Let $\delta_{S} \subset \partial S$ be a set of boundary components, and let $f_{S}: \delta_{S} \rightarrow \gamma$ be an immersion, such that the degree of $f_{S}$ on every component is positive. Let $n_{i}$ be the degrees of $f_{S}$ on the components of $\delta_{S}$, and let $N$ be a common multiple of the $n_{i}$. Then there is a finite cover $\widehat{S}$ such that every component of the preimage $\hat{\delta}_{S}$ maps to $\gamma$ with degree $N$.

Proof An orientable surface $S$ with genus at least 1 admits a double cover $S^{\prime}$ such that every component of $\partial S$ has exactly two preimages in $S^{\prime}$. Let $\delta_{S, i}$ be the components of $\delta_{S}$, and let $n_{i}$ be the degrees of the map $f_{T}: \delta_{S, i} \rightarrow \gamma$. Let $N$ be a common multiple of the $n_{i}$. Define a homomorphism $\phi: \pi_{1}\left(S^{\prime}\right) \rightarrow \mathbb{Z} / N \mathbb{Z}$ as follows. For each $i$, let $\epsilon_{i, 1}, \epsilon_{i, 2}$ be the components of $\partial S^{\prime}$ in the preimage of $\delta_{S, i}$, and let $n_{i}$ be the degree of $f_{S}: \delta_{S, i} \rightarrow \gamma$. Then define $\phi\left(\epsilon_{i, 1}\right)=n_{i}$ and $\phi\left(\epsilon_{i, 2}\right)=-n_{i}$. Since $\phi\left(\partial S^{\prime}\right)=0$ in $\mathbb{Z} / N \mathbb{Z}$, the function $\phi$ extends to a (surjective) homomorphism from $\pi_{1}\left(S^{\prime}\right)$ to $\mathbb{Z} / N \mathbb{Z}$. If $\hat{S}$ is the cover corresponding to the kernel of $\phi$, then every component of $\hat{\delta}_{S}$, the preimage of $\delta_{S}$, maps to $\gamma$ with degree $N$, as desired.

Start with a pair of positive immersed surfaces with rational boundary $C_{1}$ and $C_{2}$. Since the Euler characteristics of these surfaces are negative, they admit finite covers with genus at least one. After passing to a suitable cover (provided by the claim) and gluing up pairs of boundary components which map to geodesics $\gamma$ in the common 
support of $C_{1}$ and $C_{2}$ with the same absolute degree but with opposite signs, we obtain a positive immersed surface which $C$ rationally bounds.

Remark 3.4 Compare with the proof of Theorem 3.4 of [8].

We will give a much shorter proof of this Lemma (assuming more machinery) in Section 3.3 in the special case that the ambient surface $S$ has boundary.

\subsection{Rotation quasimorphism}

Throughout this section we fix $S$, a compact oriented hyperbolic surface with boundary.

Let $\gamma$ be a homologically trivial geodesic in $S$. The geodesic $\gamma$ cuts $S$ up into connected regions $R_{i}$. For each $i$, let $\alpha_{i}$ be an oriented arc from $\partial S$ to $R_{i}$ which is transverse to $\gamma$, and let $n_{i}$ be the signed intersection of $\alpha_{i}$ with $\gamma$. Since $\gamma$ is homologically trivial by hypothesis, $n_{i}$ does not depend on the choice of $\alpha_{i}$. Geometrically, if $T$ is an oriented surface, and $f: T, \partial T \rightarrow S, \gamma$ is a smooth map, $n_{i}$ is a signed count of the preimages of a generic point in $R_{i}$.

Definition 3.5 The algebraic area enclosed by $\gamma$ is the sum

$$
\operatorname{area}(\gamma)=\sum n_{i} \cdot \operatorname{area}\left(R_{i}\right)
$$

The hyperbolic structure and the orientation on $S$ determines a discrete faithful representation $\rho: \pi_{1}(S) \rightarrow \operatorname{PSL}(2, \mathbb{R})$ unique up to conjugacy. Since $\pi_{1}(S)$ is free, this representation lifts to $\widetilde{\rho}: \pi_{1}(S) \rightarrow \widetilde{\mathrm{SL}}(2, \mathbb{R})$, where $\widetilde{\mathrm{SL}}(2, \mathbb{R})$ denotes the universal covering group of $\operatorname{PSL}(2, \mathbb{R})$. The group $\operatorname{PSL}(2, \mathbb{R})$ acts on the circle at infinity of hyperbolic space and lets us think of $\operatorname{PSL}(2, \mathbb{R})$ as a subgroup of homeo ${ }^{+}\left(S^{1}\right)$. The covering group $\widetilde{\mathrm{SL}}(2, \mathbb{R})$ is the preimage of $\operatorname{PSL}(2, \mathbb{R})$ in homeo ${ }^{+}(\mathbb{R})$.

Definition 3.6 Given $g \in \widetilde{\mathrm{SL}}(2, \mathbb{R})$, the rotation number of $g$, as defined by Poincaré, is the limit

$$
\operatorname{rot}(g)=\lim _{n \rightarrow \infty} \frac{g^{n}(0)}{n}
$$

where we think of $\widetilde{\mathrm{SL}}(2, \mathbb{R})$ as a subgroup of homeo ${ }^{+}(\mathbb{R})$ under the covering projection $\mathbb{R} \rightarrow S^{1}=\mathbb{R} / \mathbb{Z}$.

Rotation number pulls back by $\tilde{\rho}$ to define a function rot on $\pi_{1}(S)$. As is well-known, rot is a homogeneous quasimorphism on $\pi_{1}(S)$ with $D(\operatorname{rot})=1$. As a function on $\pi_{1}(S)$, the function rot depends on the choice of lift of $\rho$ to $\tilde{\rho}$. Different lifts are classified by elements of $H^{1}(S ; \mathbb{Z})$, so rot is well-defined on $\pi_{1}(S)$ modulo 
elements of $H^{1}(S ; \mathbb{Z})$, and therefore well-defined on the commutator subgroup of $\pi_{1}(S)$ independent of the choice of $\tilde{\rho}$. Though it appears to depend on the choice of hyperbolic structure on $S$, it depends only on the topology of $S$. If we need to stress the dependence of rot on $S$, we write it $\operatorname{rot}_{S}$.

Lemma 3.7 (Area is rotation number.) If $g \in \pi_{1}(S)$, and $\gamma$ is a geodesic in $S$ corresponding to the conjugacy class of $g$, there is an equality

$$
\operatorname{area}(\gamma)=2 \pi \cdot \operatorname{rot}_{S}(g) \text {. }
$$

This is proved in [5, Lemma 4.58]. For the expert, the lemma follows from the fact that the coboundary of the rotation quasimorphism is the (relative) Euler class and the Gauss-Bonnet theorem.

Note that for every nontrivial $g \in \pi_{1}(S)$, the element $\rho(g)$ is hyperbolic in $\operatorname{PSL}(2, \mathbb{R})$, and therefore fixes two points in the circle at infinity. It follows that $\operatorname{rot}(g)$ is an integer. An explicit formula for $\operatorname{rot}(g)$, in terms of an expression of $g$ as a reduced word in a standard generating set for $\pi_{1}(S)$, is given in [5, Theorem 4.62].

The functions area and rot extend linearly and continuously to $B_{1}^{H}\left(\pi_{1}(S) ; \mathbb{R}\right)$. This is obvious for the function rot and straightforward for area: if $C=\sum_{i} t_{i} g_{i}$ where the $t_{i}$ are real numbers and the $g_{i}$ are primitive conjugacy classes, let $\gamma_{i}$ be oriented geodesics in $S$ in the conjugacy classes of the $g_{i}$. The $\gamma_{i}$ cut up $S$ into regions $R_{j}$. For each $j$, let $\alpha$ be an arc from $\partial S$ to $R_{j}$ transverse to every $\gamma_{i}$, and let $s_{j}=\sum_{i} t_{i}\left(\alpha \cap \gamma_{i}\right)$ where $\cap$ denotes signed intersection number. Then $\operatorname{area}(C)=\sum_{j} s_{j} \cdot \operatorname{area}\left(R_{j}\right)$.

We can now give necessary and sufficient conditions for a rational chain in a hyperbolic surface to rationally bound an immersed subsurface.

Proposition 3.8 Let $S$ be a compact, connected, oriented hyperbolic surface with geodesic boundary. A rational chain $C$ in $B_{1}^{H}(S)$ rationally bounds a positive immersed subsurface if and only if

$$
\operatorname{scl}(C)=\operatorname{rot}_{S}(C) / 2 \text {. }
$$

Proof Given $C \in B_{1}^{H}$ and $f: T \rightarrow S$ a surface that it rationally bounds, we can replace $T$ by a pleated surface (see eg Thurston [20, Chapter 8] for an introduction to the theory of pleated surfaces) and observe that the hyperbolic area of $T$ is at least as big as $|\operatorname{area}(C)|$, with equality if and only if the map $f$ is an immersion (note that if the original map was already an immersion, then so is its pleated representative). It follows that an immersed surface is extremal, and therefore by Theorem 2.8, if a chain $C$ bounds a positive immersed surface, then $\operatorname{scl}(C)=\operatorname{area}(C) / 4 \pi$ by Gauss-Bonnet. 
Conversely, since $S$ has boundary (by assumption) and therefore $\pi_{1}(S)$ is free, any rational chain $C$ admits an extremal surface, by the Rationality Theorem from [6] (or see Theorem 4.18 of [5]). Hence $\mathrm{scl}=\operatorname{area}(C) / 4 \pi$ if and only if $C$ bounds a positive immersed surface. By Lemma 3.7 there is an equality $\operatorname{area}(C) / 4 \pi=\operatorname{rot}_{S}(C) / 2$. $\square$

In particular, the chain $\partial S$ satisfies

$$
\operatorname{scl}(\partial S)=\operatorname{area}(S) / 4 \pi=-\chi(S) / 2 .
$$

Hence the surface $S$ itself is an extremal surface for $\partial S$.

Remark 3.9 We can use this fact to give a very short proof of Lemma 3.3 in the case that the ambient surface $S$ has boundary. A rational chain $C$ in $S$ rationally bounds a positive immersed surface if and only if $\operatorname{rot}_{S}$ is extremal for $C$, ie if $\operatorname{scl}(C)=\operatorname{rot}(C) / 2$. If $C_{1}$ and $C_{2}$ rationally bound positive immersed surfaces, then

$$
\operatorname{scl}\left(C_{1}+C_{2}\right) \leq \operatorname{scl}\left(C_{1}\right)+\operatorname{scl}\left(C_{2}\right)=\operatorname{rot}\left(C_{1}\right) / 2+\operatorname{rot}\left(C_{2}\right) / 2=\operatorname{rot}\left(C_{1}+C_{2}\right) / 2 .
$$

Hence rot is extremal for $C_{1}+C_{2}$, and therefore $C_{1}+C_{2}$ rationally bounds a positive immersed surface. Of course this proof is not "really" short, since it uses the (highly nontrivial) fact that every rational chain in a free group bounds an extremal surface.

Example 3.10 Let $S$ be a once-punctured torus, with standard generators $a, b$. Let $w \in \pi_{1}(S)$ be a nontrivial commutator, and let $\gamma$ be the associated geodesic in $S$ (necessarily primitive). It is a fact that in a free group, the "standard" once-punctured torus whose boundary is a given nontrivial commutator is always extremal. When does $\gamma$ bound an immersed surface? A description of $w$ as a cyclically reduced word in $a, b, a^{-1}, b^{-1}$ determines a polygonal loop $P_{w}$ in $\mathbb{R}^{2}$ with vertices contained in the integer lattice, as follows. Start at the origin and read the letters of $w$ one by one. On reading $a$ (resp. $a^{-1}$ ), take one step to the right (resp. left), and on reading $b$ (resp. $b^{-1}$ ), take one step up (resp. down). The polygonal loop $P_{w}$ can be "smoothed" by rounding the corners where a horizontal and a vertical arc of $P_{w}$ meet, giving rise to an immersed loop $p_{w}$. It turns out that $\gamma$ bounds an immersed surface in $S$ if and only if the winding number of $p_{w}$ is \pm 1 . So for example, $\left[a^{n}, b^{m}\right]$ bounds an immersed surface when $n, m \neq 0$, but $\left[a, b a^{-1} b^{-1}\right]$ does not. For details see Section 4.2 of [5].

\subsection{Immersion theorem}

We now prove our main technical result (Theorem C) and deduce Theorem A and Theorem B as corollaries. 
Theorem C Let $S$ be a compact, connected orientable surface with $\chi(S)<0$ and $C=\sum r_{i} g_{i}$ a finite rational chain in $B_{1}^{H}\left(\pi_{1}(S)\right)$. Then for all sufficiently large rational numbers $R$ (depending on $C$ ), the geodesic 1-manifold in $S$ corresponding to the chain $R \partial S+\sum r_{i} g_{i}$ rationally bounds an immersed surface $f: T \rightarrow S$.

Proof Since $\partial S$ bounds the immersed surface $S$, it suffices to prove the theorem for a particular positive $R$ (depending on $C$ ).

Multiply through by a large positive integer to clear denominators, so we can assume the $r_{i}$ are all integers. Also, by replacing $g_{i}$ with $g_{i}^{-1}$ if necessary, we can assume the $r_{i}$ are all positive. Pick a hyperbolic structure on $S$, and let $\gamma_{i}$ be the geodesic loop corresponding to the conjugacy class of $g_{i}$. By Scott [19] there is a finite cover of $S$ in which every component of the preimage of each $\gamma_{i}$ is embedded. In other words, there is a finite cover $\widetilde{S} \rightarrow S$, so that if $\widetilde{C}$ is the union in $\widetilde{S}$ of all preimages of all components of $C$, then every component of $\widetilde{C}$ is embedded (although the union typically will not be). The composition of a positive immersion with a covering map is a positive immersion, so it suffices to construct the positive immersion in $\widetilde{S}$. Hence without loss of generality we can assume that we are working in the cover, and every individual geodesic $\gamma_{i}$ is embedded (though of course the union will typically not be). Let $\alpha_{1}, \beta_{1}, \ldots, \alpha_{g}, \beta_{g}$ be a standard system of embedded geodesics which are a standard basis for $H_{1}(S ; \mathbb{Z}) / H_{1}(\partial S ; \mathbb{Z})$. For each $\gamma_{i}$ let $a_{i, j}, b_{i, j}$ be integers such that

$$
\left[\gamma_{i}\right]=\sum_{j} a_{i, j}\left[\alpha_{j}\right]+b_{i, j}\left[\beta_{j}\right]-D_{i}
$$

in homology, where $D_{i}$ is in the image of $H_{1}(\partial S ; \mathbb{Z})$. Since the entire boundary $\partial S$ is homologically trivial, the class $D_{i}$ is represented (in many different ways) as a positive sum of positively oriented boundary components of $S$.

The logic of the remainder of the argument is as follows. We will show that for each $i$, the chain

$$
C_{i}:=\gamma_{i}-\left(\sum_{j} a_{i, j} \alpha_{j}-b_{i, j} \beta_{j}\right)+\partial_{i}
$$

bounds a positive immersed surface, where $\partial_{i}$ is a positive sum of positively oriented boundary components of $S$. This will be enough to prove the theorem. For, since $C$ is homologically trivial, we have

$$
\sum r_{i} C_{i}=\sum_{i} r_{i} \gamma_{i}+R \partial S=C+R \partial S .
$$

Hence by Lemma 3.3, the chain $C+R \partial S$ bounds a positive immersed surface for sufficiently big $R$, as claimed. 
First, decompose $S$ along a union $\delta$ of embedded separating geodesics into a union of genus one subsurfaces $S_{k}$ such that $\alpha_{k}, \beta_{k}$ is a standard basis for $H_{1}\left(S_{k}\right) / H_{1}\left(\partial S_{k}\right)$. Hence (in particular), for each $k$, the geodesics $\alpha_{k}, \beta_{k}$ are simple and intersect transversely in one point. The first step is to replace each $\gamma_{i}$ by a union $\gamma_{i}^{\prime \prime}$ of geodesics, each of which is embedded and contained in a single subsurface $S_{k}$.

Claim For each $\gamma_{i}$ there is a chain $\gamma_{i}-\gamma_{i}^{\prime \prime}+\partial_{i}$ which bounds a positive immersed surface, where $\gamma_{i}^{\prime \prime}$ is a positive sum of embedded geodesics disjoint from $\delta$, and $\partial_{i}$ is a positive sum of positively oriented boundary components of $\partial S$.

We remark that either or both of $\gamma_{i}^{\prime \prime}, \partial_{i}$ might be empty in the statement of the claim above.

Proof If $\gamma_{i}$ is a component of $\delta$, then $\gamma_{i}$ cobounds a positive immersed surface (in fact, an embedded subsurface of $S$ ) together with some components of $\partial S$. After adding a sufficiently big multiple of $\partial S$ we obtain a chain of the form $\gamma_{i}+\partial_{i}$ as above which bounds an immersed positive surface, and we are done in this case.

Otherwise, $\gamma_{i} \cap \delta$ is in general position. The intersection $\gamma_{i} \cap S_{k}$ consists of a collection of arcs or a single embedded loop. Moreover, since $\gamma_{i}$ and $\delta$ are geodesic, every arc of $\gamma_{i} \cap S_{k}$ is essential. Among the components $S_{k}$, there are two which intersect $\delta$ each in a single component. Let $S_{k}$ be one such. Every arc of $\gamma_{i} \cap S_{k}$ has endpoints on this single component of $\delta$. There are two possibilities (not necessarily mutually exclusive):

(1) There is an $\operatorname{arc} v$ of $\gamma_{i} \cap \delta$ and an $\operatorname{arc} \mu$ of $\delta$ on the positive side of $v$ such that the interior of $\mu$ is disjoint from $\gamma_{i}$, and $\partial \mu=\partial v$.

(2) There are $\operatorname{arcs} v, v^{\prime}$ of $\gamma_{i} \cap \delta$ and $\operatorname{arcs} \mu, \mu^{\prime}$ of $\delta$ on the positive sides of $v, v^{\prime}$ such that the interiors of $\mu, \mu^{\prime}$ are disjoint from $\gamma_{i}$, and $\partial \mu \cup \partial \mu^{\prime}=\partial v \cup \partial v^{\prime}$.

In the first case we build a positive immersed surface with one boundary component on $\gamma_{i}$ by attaching a 1 -handle whose core is $\mu$. In the second case we build a positive immersed surface with one boundary component on $\gamma_{i}$ by attaching a 1-handle whose core is one of $\mu, \mu^{\prime}$. See Figure 4.

The result of attaching a 1 -handle with core $\mu$ to a product neighborhood of $\gamma_{i}$ produces an embedded positive surface. If some component of the boundary of this surface is homotopically trivial, it is necessarily trivial on the positive side, so we cap it off with an embedded disk. Straighten the resulting surface by an isotopy until its boundary components are geodesic. This produces a new geodesic 1 -manifold $r\left(\gamma_{i}\right)$ called a 

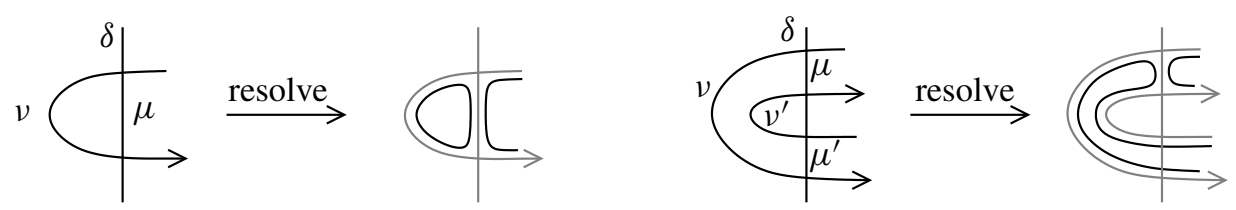

Figure 4: The 1-manifolds $\gamma_{i}$ and $-\gamma_{i}^{\prime}$ cobound a positive immersed surface, obtained from $\gamma_{i}$ by attaching a 1 -handle whose core is $\mu$.

resolution of $\gamma_{i}$ whose components are all embedded, such that $\gamma_{i}-r\left(\gamma_{i}\right)$ bounds a positive immersed surface and such that $r\left(\gamma_{i}\right)$ has at least two fewer intersections with $\delta$ than $\gamma_{i}$ does. In particular, it is certainly true that each component of $r\left(\gamma_{i}\right)$ has fewer intersections with $\delta$ than $\gamma_{i}$ does.

We resolve the components of $r\left(\gamma_{i}\right)$ in exactly the same way that we resolved $\gamma_{i}$ above, and so on, inductively. If some of the resulting components are (isotopic to) elements of $\delta$, they cobound an immersed positive surface with some positive sum of positively oriented boundary components of $\partial S$ as above.

After finitely many steps, we obtain chains $\gamma_{i}^{\prime}$ and $\partial_{i}^{\prime}$, where $\gamma_{i}^{\prime}$ is a positive sum of embedded geodesics, each disjoint from $\partial S_{k}$, where $\partial_{i}^{\prime}$ is a positive sum of positively oriented boundary components of $\partial S$, such that $\gamma_{i}-\gamma_{i}^{\prime}+\partial_{i}^{\prime}$ bounds a positive immersed surface.

Now, let $S_{k^{\prime}}$ be a component of $S-\delta$ adjacent to $S_{k}$. By construction, each component of $\gamma_{i}^{\prime}$ intersects at most one component of $\delta$ in $\partial S_{k^{\prime}}$. So the components of the $\gamma_{i}^{\prime}$ can be iteratively resolved by the method above. By induction, we end up (finally) with a chain $\gamma_{i}-\gamma_{i}^{\prime \prime}+\partial_{i}$ of the desired form, proving the claim.

Let $\epsilon$ be a component of $\gamma_{i}^{\prime \prime}$ as in the claim. Let $S_{k}$ be the component of $S-\delta$ containing $\epsilon$, and let $S^{\prime}$ be obtained from $S_{k}$ by filling in all but exactly one boundary component. For notational simplicity, denote the geodesics $\alpha_{k}, \beta_{k}$ by $\alpha, \beta$ respectively, and let $a, b$ be integers such that $[\epsilon]=a[\alpha]+b[\beta]$ in $H_{1}\left(S^{\prime}\right)$.

Since $\epsilon$ is a geodesic on $S_{k}$ but not in $S^{\prime}$, some component of $S^{\prime}-\epsilon \cup \alpha \cup \beta$ might be a bigon which contains one, or several components of $S^{\prime}-S_{k}$.

By pushing $\epsilon$ repeatedly over such components of $S^{\prime}-S_{k}$ we can eliminate such bigons, innermost first. If $\epsilon^{\prime}$ is the geodesic in $S_{k}$ obtained from $\epsilon$ by pushing $\epsilon$ over one component $\partial_{i}$ of $\partial S_{k}$, then $\epsilon$ and $\epsilon^{\prime}$ are disjoint, and either $\epsilon-\epsilon^{\prime}+\partial_{i}$ or $\epsilon-\epsilon^{\prime}+\left(\partial S_{k}-\partial_{i}\right)$ bounds a positive embedded subsurface of $S_{k}$. By gluing up finitely many such surfaces, we obtain a geodesic $\epsilon^{\prime \prime}$ in $S_{k}$, such that $\epsilon-\epsilon^{\prime \prime}$ plus some union of boundary components of $S_{k}$ bounds a positive immersed surface in $S_{k}$ and 
such that $\epsilon^{\prime \prime} \cup \alpha \cup \beta$ is in the isotopy class of a configuration of geodesics for some hyperbolic structure on $S^{\prime}$.

In this way we are reduced to arguing about embedded curves in a once-punctured torus. We will show that $\epsilon^{\prime \prime}-a \alpha-b \beta+n \partial S^{\prime}$ rationally bounds a positive immersed surface in $S^{\prime}$. If we can find such an immersed surface, then by drilling out the components of $S^{\prime}-S_{k}$ we will obtain a positive immersed surface in $S_{k}$ bounded by $\epsilon^{\prime \prime}-a \alpha-b \beta+\partial_{\epsilon}^{\prime \prime}$ for some suitable $\partial_{\epsilon}^{\prime \prime}$ which is a positive sum of boundary components of $\partial S_{k}$.

By induction, and the (well-known) classification of simple curves in a once-punctured torus, it suffices to show that the chain $a b-a-b+n[a, b]$ bounds an immersed surface in the once-punctured torus for sufficiently large $|n|$. In fact, this turns out to be true for $|n| \geq 2$. Since the algebraic area in the once-punctured torus enclosed by $a b-a-b$ is 0 , it suffices (by Lemma 3.7 and Proposition 3.8) to show that $\operatorname{scl}(2[a, b] \pm(a b-a-b))=\operatorname{scl}(2[a, b])=1$ which can be verified by calculation, eg using scallop (also see Figure 5 below).

Applying Lemma 3.3, we conclude that $\epsilon-a \alpha-b \beta+\partial_{\epsilon}$ rationally bounds a positive immersed surface, where $\partial_{\epsilon}$ is some positive sum of boundary components of $\partial S_{k}$. By adding on sufficient copies of $S-S_{k}$ we obtain a positive immersed surface with boundary $\epsilon-a \alpha-b \beta+D_{\epsilon}$, where $D_{\epsilon}$ is a positive sum of boundary components of $S$. But $\epsilon$ is an arbitrary component of $\gamma_{i}^{\prime \prime}$. By the claim and Lemma 3.3, we obtain a positive immersed surface with rational boundary $\gamma_{i}-\sum_{j} a_{i, j} \alpha_{j}-b_{i, j} \beta_{j}+\partial_{i}$ for suitable $\partial_{i}$. Since $i$ was arbitrary, this proves the theorem.

Remark 3.11 Notice that we do not assume that $\partial S$ is nonempty, just that $\chi(S)<0$. It is only the subsurfaces $S_{k}$ which are required to have nonempty boundary, which will be the case, since each $S_{k}$ has genus 1 , and $\chi(S)<0$ implies that the genus of $S$ is at least 2 .

By the results of Section 3.3 and Section 2.4 we conclude:

Theorem A Let $F$ be a free group, and let $S$ be a compact, connected, orientable surface with $\chi(S)<0$ and $\pi_{1}(S)=F$. Let $\partial S \in B_{1}^{H}(F ; \mathbb{R})$ be the 1-chain represented by the boundary of $S$, thought of as a finite formal sum of conjugacy classes in $F$. Then the projective ray in $B_{1}^{H}(F ; \mathbb{R})$ spanned by $\partial S$ intersects the unit ball of the scl norm in the interior of a face of codimension one in $B_{1}^{H}(F ; \mathbb{R})$.

Theorem B Let $F$ be a free group, and let $S$ be a compact, connected, orientable surface with $\chi(S)<0$ and $\pi_{1}(S)=F$. Let $\pi_{S}$ be the face of the scl unit norm ball whose interior intersects the projective ray of the class $\partial S$. The face $\pi_{S}$ is dual to the extremal homogeneous quasimorphism $\operatorname{rot}_{S}$. 
Remark 3.12 For the sake of completeness, we exhibit a positive immersed surface with rational boundary $2[a, b]+a+b-a b$ in Figure 5 .

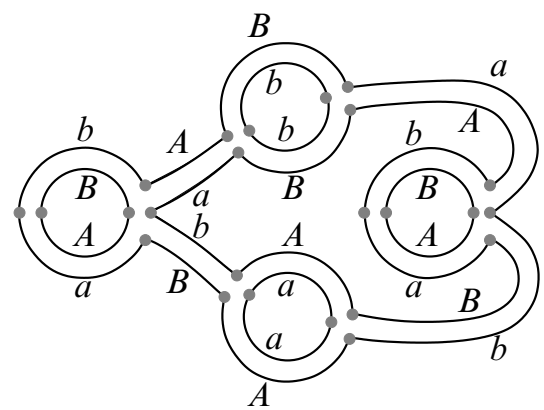

Figure 5: A positive immersed surface with rational boundary the chain $2[a, b]+a+b-a b$

The figure depicts a genus 0 surface $T$ with 6 boundary components. The boundary components are (cyclically) labeled by words in $F_{2}$ (for clarity, $A$ and $B$ are used in place of $a^{-1}$ and $b^{-1}$ ). The components are decomposed into arcs each labeled by a letter, such that adjacent arcs have opposite labels. It follows that there is a (unique) homotopy class of map $f: T \rightarrow S$ where $S$ is a once-punctured torus with standard generators $a, b$ for $\pi_{1}$ taking each boundary component of $T$ to the geodesic in $S$ corresponding to the conjugacy class of the boundary label. Two components of $\partial T$ each wrap twice around $[a, b]$ (the boundary of $S$ ). Two other components of $T$ wrap once each around $(a b)^{-1}$. One component of $T$ wraps twice around $a$, and one component wraps twice around $b$. Hence $\partial T$ represents the chain $2[a, b]^{2}+a^{2}+b^{2}-2 a b$. Since $-\chi(T) / 2=2=\operatorname{scl}\left(2[a, b]^{2}+a^{2}+b^{2}-2 a b\right)$, the homotopy class of $f$ is represented by an immersion.

\subsection{Remarks and Corollaries}

In this section we collect some miscellaneous remarks and corollaries of our main theorems. The first remark is that one can give a new proof of the relative version of rigidity theorems of Goldman [11] and Burger-Iozzi-Wienhard [3] (also compare Matsumoto [16]) about representations of surface groups with maximal Euler class.

The context is as follows. Let $S$ be a compact oriented surface with boundary, and let $\rho: \pi_{1}(S) \rightarrow \operatorname{Sp}(2 n, \mathbb{R})$ be a symplectic representation for which the conjugacy classes of boundary elements fix a Lagrangian subspace. In this case, there is a well-defined relative Euler class $\mathrm{eu}_{\rho}$ in $H^{2}(S, \partial S ; \mathbb{Z})$ associated to $\rho$. It is well-known in this context that $\mathrm{eu}_{\rho}$ is a bounded cohomology class and satisfies $\left|\mathrm{eu}_{\rho}([S])\right| \leq-\chi(S) \cdot n$. 
Our methods give a surprisingly short new proof of the following theorem (due to Goldman for $n=1$ and Burger-Iozzi-Wienhard for $n \geq 1$ ). For simplicity we restrict to Zariski dense representations; this restriction can be removed by analyzing various cases, but since the main virtue of our alternate argument is its brevity, it is probably not worth spelling out the details.

Corollary 3.13 Let $S$ be a compact oriented surface with boundary. Let $\rho: \pi_{1}(S) \rightarrow$ $\operatorname{Sp}(2 n, \mathbb{R})$ be Zariski dense, and suppose that boundary elements fix a Lagrangian subspace (so that the relative Euler class $\mathrm{eu}_{\rho}$ is defined). If $\mathrm{eu}_{\rho}([S])$ is maximal, $\rho$ is discrete.

Proof In what follows, denote $\pi_{1}(S)$ by $G$ and its commutator subgroup by $G^{\prime}$. Since $S$ has boundary, eu $\mathrm{e}_{\rho}=\delta \phi_{\rho}$ where $\phi_{\rho}$ is a homogeneous quasimorphism on $G$, unique up to elements of $H^{1}(S ; \mathbb{R})$. For each $g \in G$, the value of $\phi_{\rho}(g) \bmod \mathbb{Z}$ is the symplectic rotation number of $\rho(g)$. The symplectic rotation number lifts to a quasimorphism on the universal cover of $\operatorname{Sp}(2 n, \mathbb{R})$ with defect $n$. On the other hand, $\left|\phi_{\rho}(\partial S)\right|=\left|\mathrm{eu}_{\rho}([S])\right|=-\chi(S) \cdot n$ so we can conclude that the defect of $\phi_{\rho}$ on $G$ is exactly $n$, and $\phi_{\rho}$ is extremal for $\partial S$. Hence by Theorem B we conclude that the symplectic rotation number of every element of $\rho\left(G^{\prime}\right)$ is zero, and therefore (in particular) $\rho\left(G^{\prime}\right)$ is not dense in $\operatorname{Sp}(2 n, \mathbb{R})$. Since $\operatorname{Sp}(2 n, \mathbb{R})$ is simple, every Zariski dense subgroup is either discrete or dense (in the ordinary sense). If $\rho(G)$ is dense, then the closure of $\rho\left(G^{\prime}\right)$ is normal in $\operatorname{Sp}(2 n, \mathbb{R})$. $\operatorname{But} \operatorname{Sp}(2 n, \mathbb{R})$ is simple, and the closure of $\rho\left(G^{\prime}\right)$ is a proper subgroup; hence $\rho(G)$ is discrete.

Bavard [1] asked whether scl takes values in $\frac{1}{2} \mathbb{Z}$ in a free group. Though this turns out not to be the case, nevertheless, elements with values in $\frac{1}{2} \mathbb{Z}$ are very common. Theorem $\mathrm{C}$ gives a flexible method to construct many elements in free groups with scl in $\frac{1}{2} \mathbb{Z}$. For example, from Lemma 2.4 we conclude:

Corollary 3.14 Let $F_{2}$ denote the free group on two generators $a, b$ and let $F_{3}=$ $F_{2} *\langle c\rangle$ be a free group on three generators. For any $w \in\left[F_{2}, F_{2}\right]$ and for all integers $n$ with $|n|$ sufficiently large (depending on $w$ ) there is an equality

$$
\operatorname{scl}_{F_{3}}\left([a, b]^{n} c w c^{-1}\right)=\frac{|n+\operatorname{rot}(w)|+1}{2} \in \frac{1}{2} \mathbb{Z} .
$$

Obviously this construction can be varied considerably.

Remark 3.15 Computer experiments (using scallop) suggest that for any $w \in$ $\left[F_{2}, F_{2}\right]$, the geodesic corresponding to the conjugacy class of $w[a, b]^{n}$ rationally 
bounds an immersed surface for sufficiently large $n$. This can be proved directly for many specific elements $w$, but a general argument is lacking. Therefore we make the following conjecture:

Conjecture 3.16 Let $w \in\left[F_{2}, F_{2}\right]$ be arbitrary. Then for sufficiently large integers $n$, there is an equality

$$
\left.\operatorname{scl}\left(w[a, b]^{n}\right)=\operatorname{rot}_{S}\left(w[a, b]^{n}\right]\right) / 2
$$

where $\operatorname{rot}_{S}$ is the rotation quasimorphism associated with the realization of $F_{2}$ as $\pi_{1}(S)$ where $S$ is a hyperbolic once-punctured torus.

This certainly holds for many $w$. On the other hand, it is worth remarking that the projective classes of such chains $w[a, b]^{n}$ are necessarily in the boundary of the face $\pi_{S}$ of the scl norm ball.

One can also deduce interesting corollaries for chains in closed surface groups:

Corollary 3.17 Let $S$ be a closed, orientable surface with $\chi(S)<0$. Any rational chain $C \in B_{1}^{H}\left(\pi_{1}(S) ; \mathbb{R}\right)$ rationally bounds an injective surface.

Proof By Theorem $C$ the chain $C$ rationally bounds a positive immersed surface in $S$. But an immersion between hyperbolic surfaces with geodesic boundary is necessarily $\pi_{1}$-injective.

Remark 3.18 Theorem $\mathrm{C}$ unfortunately does not settle the question of whether scl is rational in closed (orientable) surface groups. A positive immersed surface $T$ with rational boundary a chain $C \in B_{1}^{H}(S)$ is extremal in its relative homology class, but the set of relative homology classes with boundary $C$ is a torsor for the group $\mathrm{H}_{2}(S ; \mathbb{Z})$, which is infinite when $S$ is closed and orientable. One can translate this into an absolute statement about scl at slight cost.

Let $S$ be a closed hyperbolic surface, and let $M$ be the unit tangent bundle of $S$. The Euler class of $M$, thought of as an oriented circle bundle over $S$, is an element of $H^{2}(S ; \mathbb{Z})$ whose evaluation on the fundamental class of $S$ is $\chi(S)$. The fundamental group of $M$ is a central extension

$$
0 \rightarrow \mathbb{Z} \rightarrow \pi_{1}(M) \rightarrow \pi_{1}(S) \rightarrow 0
$$

corresponding to the Euler class in $H^{2}(S ; \mathbb{Z})$. Given $g \in\left[\pi_{1}(S), \pi_{1}(S)\right]$ there are many distinct lifts $\hat{g}$ to $\pi_{1}(M)$ which differ by elements of $\chi(S) \cdot \mathbb{Z}$. Theorem $\mathrm{C}$ implies that for all but finitely many lifts, $\operatorname{scl}_{\pi_{1}(M)}(\hat{g}) \in \frac{1}{2} \mathbb{Z}$ and $\hat{g}$ is represented by a loop which rationally bounds an extremal surface whose projection to $S$ is an immersion. 
The next corollary is a purely group-theoretic statement about homologically trivial elements in closed surface groups.

Corollary 3.19 Let $G$ be a closed, orientable surface group with negative Euler characteristic, and $g \in[G, G]$. Then there is a free subgroup $F$ of $G$ and elements $g_{i}$ which are conjugates of positive powers of $g$ such that every $g_{i} \in F$, and the product of the $g_{i}$ is in $[F, F]$.

Remark 3.20 Corollary 3.19 can be thought of as saying that a homologically trivial loop in a closed surface is covered by a homologically trivial 1-manifold in some surface of infinite index. One can ask to make this result sharper:

Question 3.21 Let $G$ be a closed, orientable surface group with negative Euler characteristic, and $g \in[G, G]$. Is there a free subgroup $F$ of $G$ such that $g \in[F, F]$ ?

One can ask an analogous question for any group $G$. This question is especially interesting when $G=\pi_{1}(M)$ where $M$ is a closed 3-manifold.

Finally, we can use Theorem $\mathrm{C}$ to construct injective closed surface groups representing homology classes in certain graphs of groups. The following is the analogue of the main theorem from [8].

Corollary 3.22 Let $G$ be a graph of free and closed orientable surface groups with $\chi<0$ amalgamated along cyclic subgroups, and let $A$ be a homology class in $H_{2}(G ; \mathbb{Q})$. Let $S_{i}$ be the closed surface vertex subgroups and $r_{1}, \ldots, r_{n}$ any rational numbers with all $\left|r_{i}\right|$ sufficiently large. Then the class $A+r_{1} S_{1}+r_{2} S_{2}+. .+r_{n} S_{n}$ is rationally represented by a closed surface subgroup of $G$.

Proof This follows immediately by the argument of Theorem 3.4 from [8] and Theorem C.

It is natural to wonder whether every rational chain in $B_{1}^{H}(F)$, where $F$ is a free group, is projectively contained in the interior of a face of the scl norm of finite codimension, but in fact this is not the case, as the following example shows.

Example 3.23 By Bavard duality, the codimension of the face whose interior contains the projective class of a rational chain $C$ is one less than the dimension of the space of extremal quasimorphisms for $C\left(\bmod H^{1}\right)$. Hence to exhibit a rational chain (in fact, an element of $[F, F]$ ) which is in the interior of a face of infinite codimension, 
it suffices to exhibit a chain which admits an infinite dimensional space of extremal quasimorphisms.

Let $F=F_{1} * F_{2}$ where $F_{1}$ and $F_{2}$ are both free of rank at least 2, and let $g \in\left[F_{1}, F_{1}\right]$ be nontrivial. Let $\phi_{1} \in Q\left(F_{1}\right)$ be extremal for $g$, and let $\phi_{2} \in Q\left(F_{2}\right)$ be arbitrary with $D\left(\phi_{2}\right) \leq D\left(\phi_{1}\right)$. By the Hahn-Banach theorem, there exists $\phi \in Q(F)$ which agrees with $\phi_{i}$ on $F_{i}$ and satisfies $D(\phi)=D\left(\phi_{1}\right)$.

Another (more direct) way to see that $g$ is contained in a face of infinite codimension is as follows. Let $h$ be a nontrivial element of $\left[F_{2}, F_{2}\right]$. Let $X_{i}$ be a $K\left(F_{i}, 1\right)$ (eg a wedge of two circles) for $i=1,2$. Let $X$ be a $K(F, 1)$ obtained by joining $X_{1}$ to $X_{2}$ by an edge $e$. Let $f: S \rightarrow X$ be extremal for the chain $g^{n}+h$. If $S$ maps over $e$, we can compress $S$ along the preimage of a generic point on $e$, reducing $-\chi^{-}(S)$, which is absurd since $S$ is extremal. Hence $S$ consists of two surfaces $S_{1}, S_{2}$ one of which is extremal for $g^{n}$ in $X_{1}$, and one of which is extremal for $h$ in $X_{2}$. Hence

$$
\operatorname{scl}_{F}(n g+h)=\operatorname{scl}_{F_{1}}\left(g^{n}\right)+\operatorname{scl}_{F_{2}}(h)=\operatorname{scl}_{F_{1}}\left(g^{n}\right)+\operatorname{scl}_{F_{2}}\left(h^{-1}\right)=\operatorname{scl}_{F}(n g-h)
$$

so $g$ is not in the interior of a top face of the scl norm restricted to the vector subspace of $B_{1}^{H}(F)$ spanned by $g$ and $h$.

Acknowledgments I would like to thank Marc Burger, Benson Farb, David Fisher, Étienne Ghys, Bill Goldman, Walter Neumann, Cliff Taubes and Anna Wienhard for their comments. I am also very grateful to the anonymous referee for corrections and thoughtful comments. While writing this paper I was partially funded by NSF grant DMS 0707130.

\section{References}

[1] C Bavard, Longueur stable des commutateurs, Enseign. Math. (2) 37 (1991) 109-150 MR1115747

[2] S Blank, Extending immersions of the circle, $\mathrm{PhD}$ thesis, Brandeis University (1967)

[3] M Burger, A Iozzi, A Wienhard, Surface group representations with maximal Toledo invariant, C. R. Math. Acad. Sci. Paris 336 (2003) 387-390 MR1979350

[4] D Calegari, scallop, Computer program Available at http:// www.its.caltech.edu/ dannyc

[5] D Calegari, scl, Monograph Available at http://www.its.caltech.edu/ $\sim$ dannyc/scl/toc.html

[6] D Calegari, Stable commutator length is rational in free groups arXiv:0802.1352 
[7] D Calegari, Universal circles for quasigeodesic flows, Geom. Topol. 10 (2006) 22712298 MR2284058

[8] D Calegari, Surface subgroups from homology, Geom. Topol. 12 (2008) 1995-2007 MR2431013

[9] G B Dantzig, Linear programming and extensions, Princeton Univ. Press (1963) MR0201189

[10] G K Francis, Spherical curves that bound immersed discs, Proc. Amer. Math. Soc. 41 (1973) 87-93 MR0321112

[11] W Goldman, Discontinuous groups and the Euler class, PhD thesis, UC Berkeley (1980)

[12] M Gromov, Volume and bounded cohomology, Inst. Hautes Études Sci. Publ. Math. (1982) 5-99 (1983) MR686042

[13] A Grothendieck, Sketch of a Programme Available at http:// people.math.jussieu.fr/ leila/grothendieckcircle/mathtexts.php

[14] PB Kronheimer, T S Mrowka, Scalar curvature and the Thurston norm, Math. Res. Lett. 4 (1997) 931-937 MR1492131

[15] S Mac Lane, Homology, Classics in Math., Springer, Berlin (1995) MR1344215 Reprint of the 1975 edition

[16] S Matsumoto, Some remarks on foliated $S^{1}$ bundles, Invent. Math. 90 (1987) 343-358 MR910205

[17] J Milnor, A concluding amusement: symmetry breaking, from: "Collected papers of John Milnor, III: Differential topology”, Amer. Math. Soc. (2007) xvi+343 MR2307957

[18] P Ozsváth, Z Szabó, Link Floer homology and the Thurston norm, J. Amer. Math. Soc. 21 (2008) 671-709 MR2393424

[19] P Scott, Subgroups of surface groups are almost geometric, J. London Math. Soc. (2) 17 (1978) 555-565 MR0494062

[20] W P Thurston, The geometry and topology of three-manifolds, Princeton Univ. Math. Dept. Lecture Notes (1979) Available at http://msri.org/publications/books/ gt $3 \mathrm{~m} /$

[21] W P Thurston, A norm for the homology of 3-manifolds, Mem. Amer. Math. Soc. 59 (1986) i-vi and 99-130 MR823443

Department of Mathematics, Caltech, Pasadena, CA 91125, USA

dannyc@its.caltech.edu

http://www.its. caltech.edu/ dannyc

Proposed: Benson Farb

Received: 22 July 2008

Seconded: Dmitri Burago, Leonid Polterovich

Revised: 19 January 2009 